\title{
Erwerbsminderungsrente nach Bezug von ALG II: Auswirkungen der Refor- men auf die Versicherungsbiografien
}

\author{
Die Einführung des Arbeitslosengeldes II (ALG II) erweiterte den Kreis der Versicherten \\ der Gesetzlichen Rentenversicherung. Der Beitrag zeigt typische Versicherungsbiografien \\ aus Rentenkonten mit Zeiten des ALG-II-Bezugs, wobei Erwerbsminderungsrente häufig \\ auf ALG-II-Bezug folgt. Besonders in Westdeutschland erweist sich ein großer Anteil von \\ Versicherungsbiografien als lückenhaft, früh von Krankheit geprägt und daher mit gerin- \\ gen Anwartschaften. Die sehr niedrige Rente der vormals Langzeitarbeitslosen spiegelt die \\ Folgen ihrer schwachen Integration in die Sozialversicherung im Verlauf der Erwerbs- \\ biografien wider. Die geringen Ausgleichszahlungen an die Sozialversicherung für Zeiten \\ der Langzeitarbeitslosigkeit tragen gleichfalls zu diesem Armutsrisiko bei.
}

TATJANA MIKA, JANINE LANGE, MICHAEL STEGMANN

\section{Einleitung}

Die Hartz-IV-Gesetzgebung und die nachfolgenden Reformen des neu geschaffenen Arbeitslosengeldes II erzeugten Gewinner und Verlierer im System der Gesetzlichen Rentenversicherung (GRV). Diese Spaltung erklärt sich aus den unterschiedlichen Biografieverläufen, die sich vor Inkrafttreten der Hartz-Reformen herausgebildet hatten. Gewinner sind Rentenversicherte sowie heutige und künftige Rentnerinnen und Rentner, die in den Jahren unmittelbar vor der Einführung des ALG II nur noch selten und in geringem Umfang oder gar nicht sozialversicherungspflichtig erwerbstätig gewesen waren und aus diesem Grund keinen Anspruch auf Arbeitslosenhilfe gehabt hatten, ab 2005 aber bedürftig im Sinne des ALG II waren. Ab Januar 2005 wurden für viele dieser Personen aufgrund der Überführung der Sozialhilfe in das Arbeitslosengeld II - oft nach vielen Jahren erstmals - wieder Beiträge in die Rentenversicherungskonten gezahlt. Nach sechs Monaten des Bezugs von ALG II waren dann die Voraussetzungen für eine medizinische Rehabilitation, nach 36 Monaten die Voraussetzungen für eine Erwerbsminderungsrente (Deutsche Rentenversicherung Bund 2011) erfüllt. Verlierer der Reform waren dagegen die meisten der übergeleiteten vormaligen Bezieher von Arbeitslosenhilfe, auch wenn sie nach wie vor leistungsberechtigt waren, weil die Bezugsgröße für die Rentenbeiträge reduziert wurde. Damit fiel ab 2005 sukzessive der zukünftige Anspruch auf Rente geringer aus, als wenn das alte System der
Arbeitslosenhilfe beibehalten worden wäre. Seit 2011 sind die Beiträge zur Gesetzlichen Rentenversicherung für Zeiten des ALG-II-Bezugs ganz gestrichen worden. Der folgende Beitrag zeigt, wie groß die Gruppe der Gewinner und die der Verlierer der Reform von 2005 unter den Versicherten und Rentnern ist. Hierfür werden die Versichertenbiografien zum Stand Ende 2011 und die erstmals gezahlten Renten des Jahres 2010 untersucht. Damit werden die Auswirkungen der Hartz-IV-Reformen auf die Absicherung der Betroffenen in der Gesetzlichen Rentenversicherung empirisch nachgezeichnet.

2. Rentenrechtliche Änderungen durch Reformen des SGB II - 2005 bis 2011

Die rentenrechtliche Behandlung von Zeiten der Arbeitslosigkeit hat sich in den letzten vier Dekaden des Öfteren geändert. Die Bewertung der Arbeitslosigkeit ${ }^{1}$ in der

Arbeitslosigkeit schließt in dieser Definition alle Zeiten des Bezugs von Arbeitslosengeld, Arbeitslosenhilfe, Schlechtwettergeld und Insolvenzgeld ein, auch wenn keine Verpflichtung besteht, dem Arbeitsmarkt zur Verfügung zu stehen. Diese Definition orientiert sich am SGB VI (Sozialgesetzbuch, Sechstes Buch) und nicht am SGB II oder SGB III. 
Gesetzlichen Rentenversicherung wurde tendenziell bereits bei Reformen der bedürftigkeitsgeprüften Arbeitslosenhilfe ab dem Jahr 2000 verschlechtert, als der Bezug auf $80 \%$ des vorangegangenen sozialversicherungspflichtigen Entgelts aufgegeben wurde. Immerhin war die Höhe der Beiträge zwischen 2000 und 2004 aber noch an die Arbeitslosenhilfeleistung und damit mittelbar an das vorherige Erwerbseinkommen gekoppelt. Andererseits wurde bis 2005 von den Trägern der Sozialhilfe nur sehr selten von der Option nach $\$ 14$ BSHG (Bundessozialhilfegesetz) Gebrauch gemacht, Beiträge an die Rentenversicherung zu zahlen, was in Zeiten der Arbeitslosigkeit die Anwartschaften der Sozialhilfeempfänger erhöht hätte.

Mit der Hartz-IV-Reform wurde diese Logik grundlegend verändert (Knickrehm 2008). Im Jahr 2005 wurde zunächst ein einheitlicher Beitrag für ALG-II-Beziehende von $78 €$ monatlich festgelegt. Ab 2007 wurde dieser Betrag dann auf $40 €$ pro Monat abgesenkt. Seit dem Jahr 2011 werden für ALG-II-Beziehende gar keine Beiträge zur Rentenversicherung mehr geleistet. Damit steigen die Rentenanwartschaften für gesetzliche Renten nun in Zeiten der Arbeitslosigkeit nach Auslaufen des Arbeitslosengeldes nicht mehr. Der zunächst geringe und dann abgeschaffte Beitrag für Zeiten des ALGII-Bezugs war für sich genommen von sehr geringem direktem Wert für die Höhe der späteren Rente. Es gibt allerdings noch einen weiteren Wirkmechanismus. Damit man überhaupt eine Rente aus der Gesetzlichen Rentenversicherung beziehen kann, ist es notwendig, dass man sogenannte Wartezeiten erfüllt hat. Eine Wartezeit ist dann erfüllt, wenn eine bestimmte Anzahl an Monaten mit rentenrechtlichen Zeiten vorliegt. Die aktuelle Regelung (keine Beitragszahlungen an die GRV) stellt deshalb hinsichtlich der Erfüllung von Wartezeiten für die Gewährung einer Rente für ALG-II-Bezieher eine deutliche Verschlechterung dar, während die ursprüngliche Hartz-IV-Reform für einige eine Verbesserung durch die Bewertung der Zeit als Beitragszeit in der Gesetzlichen Rentenversicherung gebracht hatte.

Die Beiträge aus ALG II zählten für die Wartezeit der Erwerbsminderungs- und Altersrenten und auch für die medizinische Rehabilitation. Die ALG-II-Bezieher mussten zunächst keine vorzeitigen Altersrenten in Anspruch nehmen, wenn diese mit Abschlägen verbunden waren. Erwerbsminderungsrenten dagegen mussten bei Vorliegen der Voraussetzungen beantragt werden, obwohl auch diese mit Abschlägen wegen vorzeitiger Inanspruchnahme einhergehen.

Die Voraussetzungen einer Erwerbsminderungsrente sind insofern restriktiver als diejenigen der Altersrente, da eine Mindestanzahl von Beiträgen in den Lebensjahren vorliegen muss, die dem Eintritt der ärztlich bestätigten gesundheitlichen Einschränkungen unmittelbar vorausgehen. Überwiegen die Lücken in der Versicherungsbiografie, beispielsweise durch eine selbstständige Erwerbstätigkeit, einen nicht versicherungspflichtigen Minijob oder das Entfallen der ALGII-Ansprüche aufgrund eines zu hohen Haushaltseinkommens, dann bleibt nur noch die Option - und auch das nur für dauerhaft voll Erwerbsgeminderte - , die Grundsicherung bei Erwerbsminderung in Anspruch zu nehmen und erst später eine gesetzliche Altersrente zu beantragen. Die HartzIV-Reform hat die Erwerbsminderungsrente daher ab 2005 für einige erst erreichbar gemacht, die zuvor viele Jahre keine Beiträge aus sozialversicherungspflichtiger Beschäftigung oder Arbeitslosengeld auf ihrem Rentenkonto verbuchen, dann aber drei Jahre Beitragszeiten durch ALG II sammeln konnten. Der Beitrag untersucht, wie viele langjährige Bezieherinnen und Bezieher von ALG II im Jahr 2010 erstmals eine Erwerbsminderungsrente erhielten.

Eine Rente wegen Erwerbsminderung wird nur gewährt, wenn der Versicherte in den letzten fünf Jahren vor Eintritt der Erwerbsminderung mindestens 36 Monate Pflichtbeiträge zur Rentenversicherung gezahlt hat. Die erforderlichen drei Jahre mit Pflichtbeiträgen können nun ab dem Jahr 2011 nicht mehr durch Zeiten des Bezugs von Arbeitslosengeld II erreicht werden. Allerdings „verlängern“ Anrechnungszeiten den Fünf-Jahres-Zeitraum, sodass Personen, die bis Dezember 2010 die erforderliche Wartezeit angesammelt hatten, diese Rechtsposition nicht verlieren, wenn sie weiter ALG II beziehen. Die Anzahl der langjährigen ALG-II-Beziehenden, die Erwerbsminderungsrente nicht mehr beantragen können, wird daher erst sukzessive im Lauf der nächsten Jahre steigen.

\section{Daten und Methoden}

Der Beitrag fokussiert auf die Lebenslaufperspektive aus dem Blickwinkel der Gesetzlichen Rentenversicherung. Verwendet werden Daten aus den Rentenversicherungskonten, die tagesgenaue biografische Informationen enthalten. Diese Daten werden für die statistische Berichterstattung zu besonderen Statistikdatenbeständen aufbereitet. Für die Analyse der Versicherungsbiografien ist dies die Versicherungskontenstichprobe (VSKT) 2011 (Deutsche Rentenversicherung Bund 2008), für den Zugang in Erwerbsminderungs- oder Altersrente der Datensatz Vollendete Versichertenleben (VVL) 2010 (Deutsche Rentenversicherung Bund 2006). Beide Datenformate enthalten die gesamte Versicherungsbiografie ab dem ersten Beitrag in das Rentenkonto. Die Versicherungskontenstichprobe ist ein jährlich aktualisiertes Panel aus allen Versicherungskonten. Für den Zeitpunkt der Ziehung werden die erreichten Anwartschaften in der GRV nach dem aktuellen Rechtsstand der Erwerbsminderungsrente für den letzten Tag des Jahres geschätzt. Dies macht die Biografien zum Stichtag vergleichbar. Die Anwartschaften werden in Entgeltpunkten ausgedrückt, wobei ein erworbener Entgeltpunkt einem durchschnittlichen Jahreseinkommen aus sozialversicherungspflichtiger Beschäftigung entspricht. Weil in den neuen Bundesländern höhere Entgeltpunkte erworben werden, die später mit einem niedrigeren Rentenwert multipliziert werden, müssen die Auswertungen zu Anwartschaften vor der Verrentung getrennt für Ost- und Westdeutschland durchgeführt werden. Die Vollendeten Versichertenleben 
ABB. 1

\section{Westdeutsche Versicherungsbiografien mit ALG-II-Zeiten*}

\begin{tabular}{lll} 
kein RV-Kontakt & AU/Krank & Geringf. Beschäftigung \\
Schule/Berufsausbildung & Alo1: Alohilfe/ALG II & Alo2: Arbeitslosengeld \\
\hline Erwerbstätig & Sonstiges
\end{tabular}
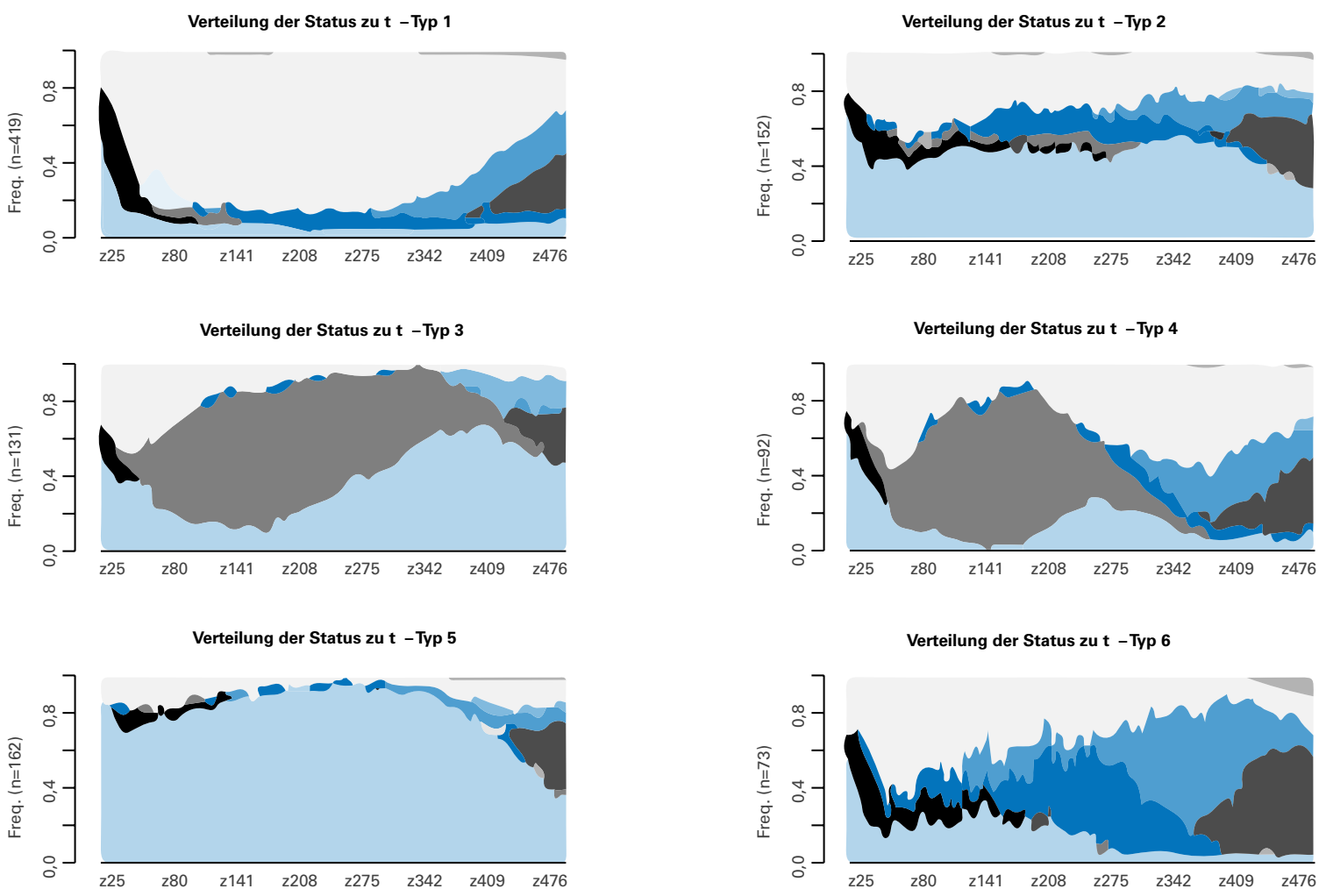

*Erwerbspersonen zwischen 16 bis 55 Jahren.

$z=$ Monat der Beobachtung: 25 = Januar des Jahres, in dem die Person 16 Jahre alt wird und 504 = Dezember des Jahres, in dem die Person 55 Jahre alt wird Zum näheren Veständnis der Arbeitslosenzeiten:

Kategorie „Alo1“: vorrangig Arbeitslosenhilfe bzw. ALG II (Arbeitslosenhilfe verlässlich erst ab dem Jahr 2001 gemeldet). Kategorie "Alo2": Arbeitslosengeldbezug und Anrechnungszeiten für Arbeitslosigkeit sowie Zeiten der Arbeitslosigkeit mit einem parallelen und prioritären anderen Sozialversicherungstatbestand.

werden in ausgewählten Jahren gezogen und umfassen jeweils $20 \%$ eines Rentenzugangsjahrgangs. In diesem Datensatz ist die gezahlte Rente enthalten. Der Beitrag untersucht dementsprechend die Versichertenbiografien im Hinblick auf die bis 2011 erreichten Anwartschaften und die im Jahr 2010 gezahlten Erwerbsminderungsrenten im Rentenzugang.

Die Versicherungsbiografien der VSKT werden explorativ daraufhin untersucht, welche Verlaufsmuster Lebensläufe zeigen, die mindestens einen Monat ALG II, in der VVL diejenigen, die mindestens 36 Monate ALG II beinhalten, bezogen haben. Dies spiegelt die rechtliche Voraussetzung für den Erhalt einer Erwerbsminderungsrente wider, da in den letzten fünf Jahren vor der Rente die drei Jahre mit Pflichtbeiträgen (hier aus ALG II) gefüllt sein müssen. Unter den langjährigen ALG-II-Beziehern wird dann die Gruppe ausgewiesen, welche im Zeitraum von 2000 bis 2004 weder Beiträge aus sozialversicherungspflichtiger Erwerbstätigkeit noch Arbeitslosigkeit aufweist und somit im System von Arbeitslosengeld und Arbeitslosenhilfe nicht abgesichert war. Personen ohne Beschäftigungszeiten vor 2005 konnten vor der Einführung von ALG II aufgrund fehlender Versicherungszeiten keine Erwerbsminderungsrente (EM-Rente) beantragen. Was die Arbeitsmarktintegration der ALG-II-Bezieher zwischen 2000 und 2004 anbelangt, werden vier Gruppen gebildet, welche nach den Kriterien unterscheiden, ob die betreffenden Personen überhaupt nicht, wenig ( 1 bis 18 Monate), teilweise (19 bis 35 Monate) oder vorwiegend (mindestens 36 Monate) sozialversicherungspflichtig erwerbstätig waren. Als Vergleichsgruppe wird zudem auf die Gruppe der Personen eingegangen, welche von 2005 bis 2010 mindestens 36 Monate an Erwerbstätigkeit aufweist. Die anschließende Analyse zeigt die Verteilung der Rentnerinnen und Rentner auf die unterschiedlichen Erwerbsbiografien und die durchschnittlichen Rentenhöhen, die ihnen ab 2010 gezahlt wurden. 
TABELLE 1

Merkmale der Biografie-Typen mit Versicherungszeiten ausschließlich in Westdeutschland

Angaben in absoluten Zahlen und in Prozent

\begin{tabular}{|c|c|c|c|c|c|c|c|c|c|}
\hline & & & $\begin{array}{l}\text { kein ALG-II- } \\
\text { Kontakt }\end{array}$ & Typ 1 & Typ 2 & Typ 3 & Typ 4 & Typ 5 & Typ 6 \\
\hline & & & $100 \%(\mathrm{~N})$ & $41 \%(419)$ & $15 \%(152)$ & $13 \%(131)$ & $9 \%(92)$ & $16 \%(162)$ & $7 \%(73)$ \\
\hline & & & Mittelwert & Mittelwert & Mittelwert & Mittelwert & Mittelwert & Mittelwert & Mittelwert \\
\hline & & $\begin{array}{l}\text { Monate mit } \\
\text { ALG-II-Bezug }\end{array}$ & 0 & 30 & 37 & - & - & 43 & 48 \\
\hline & Männlich & $\begin{array}{l}\text { Summe } \\
\text { Entgeltpunkte* } \\
\text { projiziert }\end{array}$ & 39,63 & 33,9 & 13,9 & - & - & 4,51 & 19,42 \\
\hline Geschlecht & & $\begin{array}{l}\text { Monate mit } \\
\text { ALG-II-Bezug }\end{array}$ & 0 & 31 & 41 & 40 & 31 & 43 & 30 \\
\hline & Weiblich & $\begin{array}{l}\text { Summe } \\
\text { Entgeltpunkte } \\
\text { projiziert }\end{array}$ & 21,55 & 25,57 & 11,81 & 11,07 & 20,57 & 3,03 & 21,05 \\
\hline & & $\begin{array}{l}\text { Anzahl } \\
\text { Kinder }\end{array}$ & 1,69 & 1,36 & 1 & 3,22 & 2,64 & 1,13 & 1,07 \\
\hline
\end{tabular}
den Durchschnitt ihrer Anwartschaften der Vergangenheit erwerben würden. Die Rentenanwartschaft fällt in Zukunft höher aus, wenn die in der Zukunft liegende Erwerbsbiografie besser als die vergangene verläuft.

Quelle: Versicherungskontenstichprobe (2011); Berechnungen der Autoren.

\section{Versicherungsbiografien mit Bezug von ALG II}

West- und Ostbiografien sind in unterschiedlichem Ausmaß von längeren Phasen des ALG-II-Bezugs betroffen. Von den Personen, die ihr Versichertenleben ausschließlich im Westen der Bundesrepublik verbracht haben, lassen sich bei ca. $8 \%$ auch Phasen mit ALG-II-Bezug ab dem Jahr 2005 erkennen. In Ost-Biografien, die sich zumindest teilweise im sogenannten Beitrittsgebiet abgespielt haben, tauchen in $20 \%$ der Versicherungsverläufe Zeiten des ALG-II-Bezugs auf. Die Analyse der Versicherungsverläufe, die getrennt für ost- und westdeutsche Biografien durchgeführt wurde, ergibt für Westdeutschland vielfältigere Lebenslauftypen (Abbildung 1), als sie in den ostdeutschen Biografien zu finden sind. Zwei Lebenslauf-Typen in Westdeutschland speisen sich ausschließlich aus den Lebensläufen von Frauen, während in Ostdeutschland die Biografien von Männern und Frauen so ähnlich sind, dass sie in denselben Biografie-Typen zusammengeführt werden.

In Westdeutschland finden sich insgesamt sechs Typen von Lebensläufen, die sich sehr deutlich unterscheiden. Der erste und mit $41 \%$ größte Typ zeichnet sich durch lange und kontinuierliche Erwerbsverläufe aus und weicht in der Summe der Anwartschaften dementsprechend am wenigsten von der Referenzgruppe der Personen ohne ALG-II-Zeiten ab (Tabelle 1). Die geringste Anzahl an Entgeltpunkten weisen die Typen 2, 3 und 5 auf, die jeweils jeden siebten Versicher- ten mit ALG-II-Zeiten umfassen. Damit haben fast die Hälfte der ALG-II-Bezieher nur sehr geringe Anwartschaften. Typ 2 hat erkennbare Schwierigkeiten mit Blick auf die Arbeitsmarktintegration: Dieses Problem zeigt sich hier bereits zu Beginn der Berufskarriere und ab dem mittleren Alter gibt es längere Zeiten von Krankengeld- und dann auch Arbeitslosengeld-Bezug. Typ 5 zeichnet sich dagegen dadurch aus, dass er keine relevanten Anwartschaften in der Sozialversicherung aufweisen kann. Dies sind Personen, die durch die bis 2011 geltende Pflichtversicherung über das ALG II und teilweise durch geringfügige Beschäftigung Pflichtbeiträge erworben haben, die jedoch aus der Perspektive der individuellen Alterssicherung aufgrund der kurzen Dauer und niedrigen Beitragshöhe wenig Gewicht haben. Die Anwartschaften entsprechen nur zweistelligen monatlichen Rentenzahlungen. Typ 6 umfasst nur 7 \% der Personen mit ALG-IIBezug. In diesem Typ finden sich vor allem Männer. Kennzeichnend ist auch, dass es sich hier um Personen handelt, die bereits zu Beginn der Berufskarriere Krankheitszeiten aufweisen, welche sich dann im mittleren Alter soweit verstetigen, dass die Erwerbstätigkeit immer sporadischer oder aufgegeben wird. Im Anschluss an derart ausgedehnte Krankheitsphasen treten dann die verschiedenen Formen der Arbeitslosenunterstützung an die Stelle des vorher bezogenen Krankengeldes. Im Mittel werden in solchen Fällen nur ca. 20 Entgeltpunkte erreicht, was nur einer unzureichenden Absicherung bei Erwerbsminderung sowie im Alter entspricht. Die Typen 3 und 4 bestehen ausschließlich aus Frauen. Typ 3 sind Frauen, die in der Regel früh im Leben eine 


\section{Ostdeutsche Versicherungsbiografien mit ALG-II-Zeiten*}

\begin{tabular}{lll} 
kein RV-Kontakt & AU/Krank & Geringf. Beschäftigung \\
Schule/Berufsausbildung & Alo1: Alohilfe/ALG II & Alo2: Arbeitslosengeld \\
\hline Erwerbstätig & Sonstiges
\end{tabular}

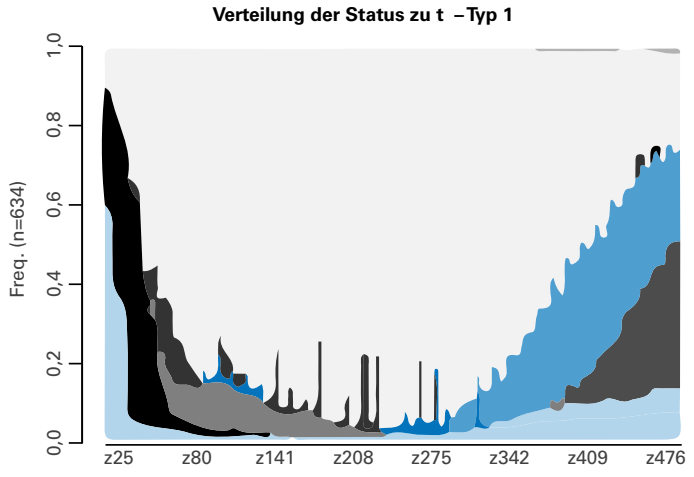

Verteilung der Status zu t - Typ 3

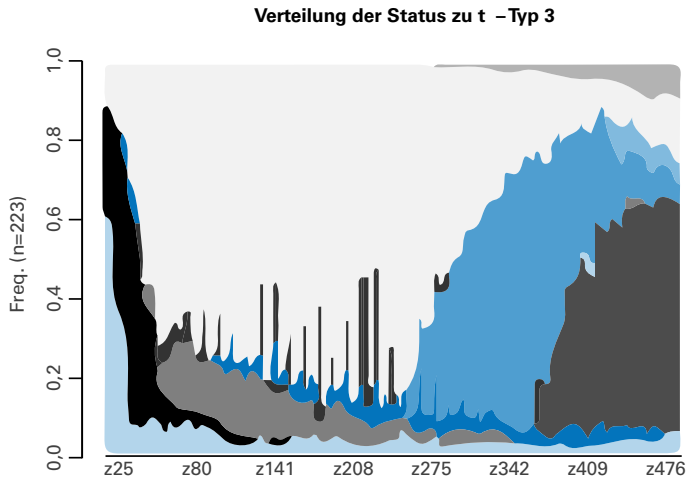

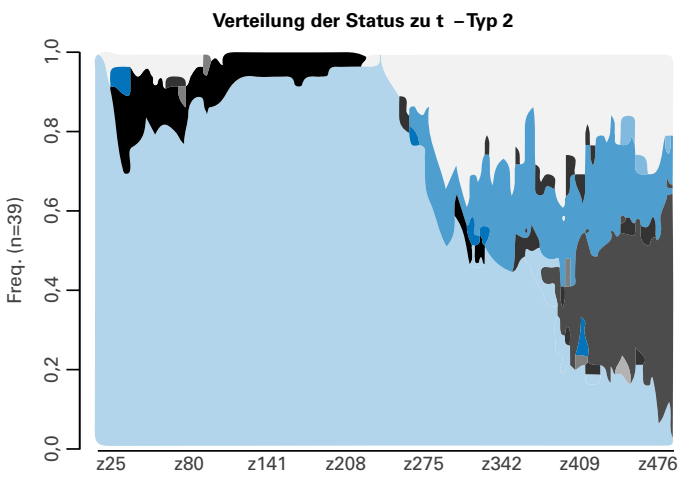

Verteilung der Status zu t - Typ 4

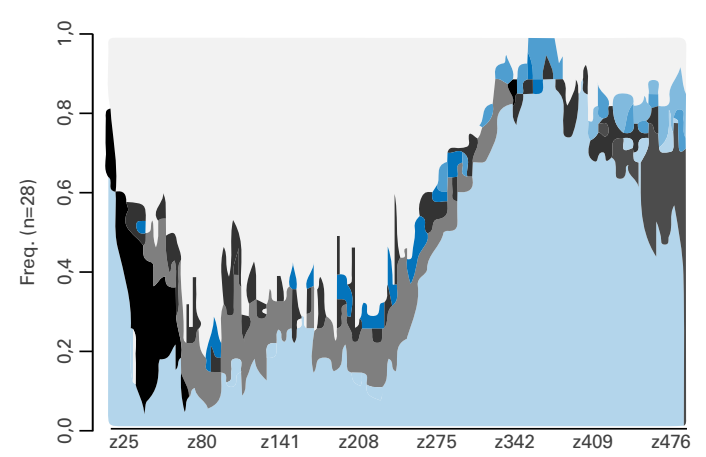

*Erwerbspersonen zwischen 16 bis 55 Jahren.

$\mathrm{z}=$ Monat der Beobachtung: 25 = Januar des Jahres, in dem die Person 16 Jahre alt wird und 504 = Dezember des Jahres, in dem die Person 55 Jahre alt wird. Zum näheren Veständnis der Arbeitslosenzeiten:

Kategorie „Alo1“: vorrangig Arbeitslosenhilfe bzw. ALG II (Arbeitslosenhilfe verlässlich erst ab dem Jahr 2001 gemeldet).

Kategorie "Alo2": Arbeitslosengeldbezug und Anrechnungszeiten für Arbeitslosigkeit sowie Zeiten der Arbeitslosigkeit mit einem parallelen und prioritären anderen Sozialversicherungstatbestand.

Familienphase beginnen, die sich rentenrechtlich als Kindererziehungszeiten abbildet. Diese Phase wird dann in der Versicherungsbiografie in erster Linie abgelöst durch geringfügige Beschäftigung oder den Bezug von ALG II, aber es gibt auch Zeiten ohne jedwede Form von Einzahlungen in die Sozialversicherung. Die Gruppe des Typs 4 hingegen beinhaltet Frauen, die häufig ebenfalls eine Phase der Familienarbeit zurückgelegt haben, aber danach wieder stärker den Weg in die Erwerbsarbeit finden. Dabei breiten sich mit zunehmendem Alter Phasen des Bezugs von Arbeitslosengeld und ALG II stärker aus.

In Ostdeutschland (Abbildung 2) lassen sich vier Typen von Personen mit ALG-II-Bezug identifizieren, wobei Typ 1 mit $69 \%$ der Personen ganz deutlich dominiert, gefolgt von Typ 3 mit $24 \%$. Dies liegt an den Spezifika der Erwerbsbiografien dieser Jahrgänge, für die in der ehemaligen DDR eine versicherungspflichtige (Vollzeit)Erwerbs- tätigkeit typisch war. Charakteristisch sind dazu auch die „kleinen Spitzen“ mit Krankheitszeiten, die aus der verwaltungstechnischen Besonderheit herrühren, dass diese jeweils am Ende der Beschäftigungsperiode im Kalenderjahr, das heißt in der Regel am Jahresende, summarisch erfasst wurden. Anders als in Westbiografien weisen diese Krankheitszeiten vor 1990 noch nicht notwendig auf schwerwiegendere dauerhafte Erkrankungen hin.

Die beiden Typen 1 und 3 unterscheiden sich insbesondere durch die unterschiedlich frühe Betroffenheit von Arbeitslosigkeit, die bei Typ 3 bereits etwa ab dem Alter von 40 Jahren einsetzt und die betroffenen Personen auf Dauer aus dem Arbeitsmarkt gedrängt hat. Bei Typ 1 sind Phasen von Arbeitslosigkeit erst später zu konstatieren und sie verdrängen die Beschäftigung nicht vollständig. Beide Typen erreichen unter anderem aufgrund der lang andauernden Arbeitslosigkeit im Mittel bei den Männern 
TABELLE 2

\section{Merkmale der Biografie-Typen mit Versicherungszeiten in Ostdeutschland}

Angaben in absoluten Zahlen und in Prozent

\begin{tabular}{|c|c|c|c|c|c|c|c|}
\hline & & & $\begin{array}{l}\text { kein ALG-II- } \\
\text { Kontakt }\end{array}$ & Typ 1 & Typ 2 & Typ 3 & Typ 4 \\
\hline & & & $100 \%(N)$ & $69 \%(634)$ & $4 \%(39)$ & $24 \%(223)$ & $3 \%(28)$ \\
\hline & & & Mittelwert & Mittelwert & Mittelwert & Mittelwert & Mittelwert \\
\hline \multirow{5}{*}{ Geschlecht } & \multirow{2}{*}{ Männlich } & $\begin{array}{l}\text { Monate mit } \\
\text { ALG-II-Bezug }\end{array}$ & 0 & 34 & 40 & 41 & 38 \\
\hline & & $\begin{array}{l}\text { Summe Entgelt- } \\
\text { punkte projiziert }\end{array}$ & 41,56 & 34,23 & 7,16 & 26,8 & 14,9 \\
\hline & \multirow{3}{*}{ Weiblich } & $\begin{array}{l}\text { Monate mit } \\
\text { ALG-II-Bezug }\end{array}$ & 0 & 27 & 42 & 39 & 29 \\
\hline & & $\begin{array}{l}\text { Summe Entgelt- } \\
\text { punkte projiziert }\end{array}$ & 34,53 & 28,87 & 6,25 & 27,32 & 14,5 \\
\hline & & Anzahl Kinder & 1,81 & 1,82 & 1,11 & 2,38 & 2,33 \\
\hline
\end{tabular}

acht Entgeltpunkte weniger als der jeweils besser gestellte Vergleichstyp ohne Bezug von ALG II. Typ 1 hat im Durchschnitt nur 34 statt 42 Entgeltpunkte und Typ 3 nur noch 26 Entgeltpunkte (Tabelle 2). Typ 2 vereinigt in sich die Personen, die erst ab dem Alter Anfang 40 durch Erwerbstätigkeit, aber auch durch Arbeitslosigkeit und dabei insbesondere aus ALG II, Anwartschaften in der Rentenversicherung aufbauen konnten. Es handelt sich hierbei um eine kleine Gruppe, überwiegend Frauen, die sehr spät mit der Sozialversicherung in Kontakt kommen und damit einen dort selten vorkommenden Lebenslauf aufweisen. Typ 4 sind dagegen Personen, bei denen es sich genau umgekehrt verhält. Bis Anfang 40 zeigt sich eine Prägung durch sozialversicherungspflichtige Erwerbstätigkeit, die dann allerdings nicht mehr fortgesetzt wurde. Bezug von ALG II tritt erst spät mit Mitte Fünfzig ein. Typ 2 und 4 haben in ihren Erwerbsbiografien gleichermaßen keine hinreichenden Zeiten sozialversicherungspflichtiger Beschäftigung, um eine befriedigende Erwerbsminderungs- und Alterssicherung aufbauen zu können. Beide zusammen umfassen etwa 7 \% der Versicherten. Im Zusammenhang mit dem ALG-II-Geschehen ist damit jener Sachverhalt zu erkennen, welchen schon Trischler und Kistler (2012) betont haben: dass sowohl Arbeitslosigkeitsphasen als auch ein insgesamt rückläufiger Anteil von Erwerbszeiten in sozialversicherungspflichtiger Beschäftigung die Erwerbsbiografie - vor allem auch im Rentenübergangsgeschehen - nachhaltig perforieren können und in der Folge in niedrigere Rentenanwartschaften einmünden. Ergebnis unserer Analyse ist, dass $44 \%$ der westdeutschen Versicherten mit ALG-II-Zeiten nur sehr geringe Anwartschaften in der Rentenversicherung aufgebaut haben und $7 \%$ der Ostdeutschen.

\section{Erwerbsminderungsrenten nach ALG-II-Bezug}

In den Daten zum Rentenzugang 2010 (VVL 2010) befinden sich insgesamt ca. 22 \% Erwerbsminderungsrentner, wobei Männer (25\%) ein wenig mehr von Erwerbsminderungsrente betroffen sind als Frauen (20\%). Bei $35 \%$ aller Erwerbsminderungsrentnerinnen und -rentner zeigt sich, dass in ihr Rentenkonto auch solche Einzahlungen eingeflossen sind, die aus einem mindestens einmonatigen ALG-II-Bezug in den Jahren 2005 bis 2010 resultieren. Diese Quote ist höher als bei Altersrentnern und Versicherten (Mika/Lange 2014). Die Erwerbsminderungsrente hat damit eine große Bedeutung als Endpunkt der Versicherungsbiografie von ALG-IIBeziehern. Dies bestärkt Forschungsergebnisse, die darauf hinweisen, dass gesundheitliche Einschränkungen, insbesondere auch psychische Erkrankungen, unter Arbeitslosen stärker verbreitet sind als unter Beschäftigten (Thielen/Kroll 2013). Außerdem ist eine eingeschränkte Gesundheit auch ein verbreitetes Motiv unter ALG-II-Beziehenden, sich den Übergang in die Rente zu wünschen (Wübbeke 2013, S. 69). Für die nachfolgenden Auswertungen wurden längere Biografiephasen im ALG-II-Bezug berücksichtigt, um diejenigen zu identifizieren, die aufgrund der Zeiten des ALG-II-Bezugs die Voraussetzungen der Erwerbsminderungsrente erfüllen konnten. Deshalb wird nur der langjährige (mindestens 36 Monate dauernde) ALG-II-Bezug untersucht, der damit die Höhe der Anwartschaften in den Jahren seit 2005 prägt. Innerhalb der Gruppe der langjährigen ALG-II-Bezieher wird dann auf der Grundlage der vorausgehenden Erwerbsbiografie klassifiziert. 


\section{Durchschnittliche Höhen der Erwerbsminderungsrenten von Frauen nach ALG-II-Bezug*}

Angaben in Euro und in Prozent

\begin{tabular}{|c|c|c|c|c|c|}
\hline & Mittelwert & $20 \%$ Perzentil & $80 \%$ Perzentil & Fallzahl (Anteil) & Anteil \\
\hline Alle EM-Renten: & $578 €$ & $343 €$ & $796 €$ & 13.814 & $100 \%$ \\
\hline $\begin{array}{l}\text { EM-Rente nach langjährigem ALG-II-Bezug (mindestens } \\
36 \text { Monate ALG-II-Bezug im Zeitraum von } 2005 \text { bis 2010) }\end{array}$ & $414 €$ & $217 €$ & $594 €$ & 2.780 & $20,1 \%$ \\
\hline unter den langjährigen ALG-II-Bezieherinnen: & & & & & $100 \%$ \\
\hline $\begin{array}{l}\text { EM-Rentnerinnen, die im Zeitraum von } 2000 \text { bis } 2004 \\
\text { weder Beiträge aus Erwerbstätigkeit noch Arbeitslosigkeit } \\
\text { aufweisen }\end{array}$ & $343 €$ & $140 €$ & $538 €$ & 925 & $33,3 \%$ \\
\hline unter den langjährigen ALG-II-Bezieherinnen: & & & & & $100 \%$ \\
\hline $\begin{array}{l}\text { EM-Rentnerinnen, die im Zeitraum von } 2000 \text { bis } 2004 \\
\text { keine Erwerbstätigkeit aufweisen }\end{array}$ & $377 €$ & $168 €$ & $572 €$ & $1.372(49,4 \%)$ & $49,4 \%$ \\
\hline $\begin{array}{l}\text { EM-Rentnerinnen, die im Zeitraum von } 2000 \text { bis } 2004 \\
1 \text { bis } 18 \text { Monate an Erwerbstätigkeit aufweisen }\end{array}$ & $434 €$ & $258 €$ & $595 €$ & $835(30 \%)$ & $30 \%$ \\
\hline $\begin{array}{l}\text { EM-Rentnerinnen, die im Zeitraum von } 2000 \text { bis } 2004 \\
19 \text { bis } 35 \text { Monate an Erwerbstätigkeit aufweisen }\end{array}$ & $471 €$ & $301 €$ & $631 €$ & $345(12,4 \%)$ & $12,4 \%$ \\
\hline $\begin{array}{l}\text { EM-Rentnerinnen, die im Zeitraum von } 2000 \text { bis } 2004 \\
\text { mindestens } 36 \text { Monate an Erwerbstätigkeit aufweisen }\end{array}$ & $481 €$ & $294 €$ & $648 €$ & $228(8,2 \%)$ & $8,2 \%$ \\
\hline \multicolumn{6}{|l|}{ Zum Vergleich: } \\
\hline $\begin{array}{l}\text { EM-Rentnerinnen, die mindestens } 36 \text { Monate Erwerbs- } \\
\text { tätigkeit im Zeitraum von } 2005 \text { bis } 2010 \text { aufweisen }\end{array}$ & $636 €$ & $394 €$ & $858 €$ & $7.405(53,6 \%)$ & $53,6 \%$ \\
\hline
\end{tabular}

*differenziert nach der Erwerbssituation vor 2005 und nach den obersten und untersten Prozent-Perzentilen

Tabelle 3 zeigt, dass jede fünfte EM-Rentnerin im Rentenzugang 2010 aus dem langjährigen ALG-II-Bezug kam. Unter ihnen hat ein Drittel in den Jahren 2000 bis 2004 weder Beiträge aus sozialversicherungspflichtiger Erwerbstätigkeit noch aus Arbeitslosigkeit an die Rentenversicherung gezahlt. Diese EM-Rentnerinnen waren mit großer Wahrscheinlichkeit Sozialhilfebezieherinnen, bevor 2005 ALG II eingeführt wurde. Etwa die Hälfte der langjährigen ALG-II-Bezieherinnen hat von 2000 bis 2004 keinen einzigen Monat in sozialversicherungspflichtiger Beschäftigung verbracht. Die Differenz zu der Gruppe der vermutlichen Sozialhilfeempfängerinnen besteht darin, dass diese Personen Beiträge aus Zeiten der Arbeitslosigkeit im Konto aufweisen, also vor dem Jahr 2000 noch erwerbstätig waren. In beiden Gruppen fällt der Rentenbetrag sehr niedrig aus. Er liegt etwa ein Drittel unter dem Durchschnitt aller EMRentnerinnen. Weil sich die Zeiten der Kindererziehung, die rentenrechtlich hoch bewertet werden, bei Erwerbsminderungsrentnerinnen besonders günstig bemerkbar machen, sind die Abstufungen zwischen den sehr unterschiedlich lange in den Arbeitsmarkt integrierten Untergruppen der späteren ALG-II-Bezieherinnen bei den Rentenbeträgen nicht sehr stark ausgeprägt. Allerdings erreicht finanziell gesehen nur die Vergleichsgruppe der langjährig ab 2005 erwerbstätigen Frauen im Durchschnitt eine Rentenhöhe, mit der der Lebensunterhalt eigenständig gesichert werden kann. Weil langjährige ALG-II-Bezieherinnen weitgehend entsparen mussten und auch im Haushaltskontext bedürftig waren, ist die Mehrheit der vormaligen ALG-II-Bezieherinnen auch nach dem Eintritt in die EM-Rente mit großer Wahrscheinlichkeit auf bedürftigkeitsgeprüfte Leistungen, also die Grundsicherung bei Erwerbsminderung, oder auf eine Aufbesserung des Haushaltseinkommens durch die Erwerbstätigkeit des Partners angewiesen. Ostdeutsche finden sich nicht überdurchschnittlich häufig unter den Erwerbsminderungsrentnerinnen. Ihre Renten sind im Durchschnitt etwas höher als die der westdeutschen Frauen, aber ebenfalls unter dem durchschnittlichen Grundsicherungsbedarf.

Tabelle 4 zeigt die entsprechenden Werte für die Männer. In der Gruppe der langjährigen ALG-II-Bezieher hat jeder achte in den Jahren 2000 bis 2004, also vor der Einführung von ALG II, keinerlei Beiträge an die Rentenversicherung entrichtet und es wurden auch keine Rentenbeiträge aus der Arbeitslosensicherung heraus gezahlt. Männer dieser Untergruppe haben noch niedrigere Renten als die Vergleichsgruppe bei den Frauen. Mit durchschnittlich $235 €$ bleibt bei ihnen die durchschnittliche Rente besonders deutlich unter dem Maßstab einer eigenständigen Sicherung des Lebensunterhalts. Andererseits hätten genau diese 
TABELLE 4

\section{Durchschnittliche Höhen der Erwerbsminderungsrenten von Männern nach ALG-II-Bezug*}

Angaben in Euro und in Prozent

\begin{tabular}{|c|c|c|c|c|c|}
\hline & Mittelwert & $20 \%$ Perzentil & $80 \%$ Perzentil & Fallzahl & Anteil \\
\hline Alle EM-Renten: & $638 €$ & $361 €$ & $898 €$ & 14.116 & $100 \%$ \\
\hline $\begin{array}{l}\text { EM-Rente nach langjährigem ALG-II-Bezug (mindestens } \\
36 \text { Monate ALG-II-Bezug im Zeitraum von } 2005 \text { bis 2010) }\end{array}$ & $416 €$ & $221 €$ & $600 €$ & 4.014 & $28,4 \%$ \\
\hline unter den langjährigen ALG-II-Beziehern: & & & & & $100 \%$ \\
\hline $\begin{array}{l}\text { EM-Rentner, die im Zeitraum von } 2000 \text { bis } 2004 \\
\text { weder Beiträge aus Erwerbstätigkeit noch Arbeitslosigkeit } \\
\text { aufweisen }\end{array}$ & $235 €$ & $77 €$ & $397 €$ & 491 & $12,2 \%$ \\
\hline unter den langjährigen ALG-II-Beziehern: & & & & & $100 \%$ \\
\hline $\begin{array}{l}\text { EM-Rentner, die im Zeitraum von } 2000 \text { bis } 2004 \\
\text { keine Erwerbstätigkeit aufweisen }\end{array}$ & $350 €$ & $160 €$ & $522 €$ & 1.404 & $35 \%$ \\
\hline $\begin{array}{l}\text { EM-Rentner, die im Zeitraum von } 2000 \text { bis } 2004 \\
1 \text { bis } 18 \text { Monate an Erwerbstätigkeit aufweisen }\end{array}$ & $401 €$ & $216 €$ & $570 €$ & 1.415 & $35,3 \%$ \\
\hline $\begin{array}{l}\text { EM-Rentner, die im Zeitraum von } 2000 \text { bis } 2004 \\
19 \text { bis } 35 \text { Monate an Erwerbstätigkeit aufweisen }\end{array}$ & $479 €$ & $297 €$ & $648 €$ & 697 & $17,4 \%$ \\
\hline $\begin{array}{l}\text { EM-Rentner, die im Zeitraum von } 2000 \text { bis } 2004 \\
\text { mindestens } 36 \text { Monate an Erwerbstätigkeit aufweisen }\end{array}$ & $561 €$ & $371 €$ & $733 €$ & 498 & $12,4 \%$ \\
\hline \multicolumn{6}{|l|}{ Zum Vergleich: } \\
\hline $\begin{array}{l}\text { EM-Rentner, die mindestens } 36 \text { Monate Erwerbs- } \\
\text { tätigkeit im Zeitraum von } 2005 \text { bis } 2010 \text { ausweisen }\end{array}$ & $773 €$ & $499 €$ & $1.018 €$ & 6.560 & $46,5 \%$ \\
\hline
\end{tabular}

*differenziert nach der Erwerbssituation vor 2005 und nach den obersten und untersten Prozent-Perzentilen.

Quelle: Deutsche Rentenversicherung Bund, VVL/RTZN (2010); Berechnungen der Autoren

(ohne Sondergruppen Beschäftigte in geschützten Werkstätten und Pflegepersonen, keine Nullrenten).

Personen bei Weiterführung des Systems der Arbeitslosenhilfe auf dem Rechtsstand von 2004 gar keine EM-Rente beziehen können, denn sie hatten keine ausreichenden Zeiten der Vorversicherung. Die größten Gruppen unter den langjährigen ALG-II-Beziehern im Zugang zur Erwerbsminderungsrente sind diejenigen, die vor 2005 gar nicht oder nur wenig, also bis zu 18 Monaten sozialversicherungspflichtig erwerbstätig waren. Gemeinsam machen sie über 70 \% aller langjährigen ALG-II-Bezieher im EM-Rentenzugang aus. Der Bezug der langjährigen ALG-II-Bezieher zum Arbeitsmarkt war also in der gesamten Dekade vor der Verrentung nur noch schwach. Nur ein Achtel von ihnen ist aus einer Phase überwiegender Erwerbstätigkeit im Zeitraum von 2000 bis 2004 heraus in ALG II und von dort in die EM-Rente gewechselt. Dies ist die Gruppe derjenigen, die durch die niedrigen Beiträge zur Rentenversicherung ab 2005 nunmehr eine geringere Rente haben, als sie ihnen bei Fortführung der Sicherung durch Arbeitslosenhilfe zugestanden hätte. Und es ist weiterhin die Gruppe derjenigen, von denen nunmehr einige infolge der Reform unter die Schwelle des Grundsicherungsbedarfs gerutscht sind.

Mehr als jeder vierte Erwerbsminderungsrentner im Rentenzugang 2010 kam aus langjährigem ALG-II-Bezug. Der Durchschnitt ihrer Renten war mit etwas mehr als $400 €$ nicht ausreichend, um davon den eigenen Lebensunterhalt zu bestreiten. Damit sind - wie bei den Frauen - auch Männer, die langjährig ALG II bezogen haben, in der Mehrheit auf aufstockende Grundsicherung bei Erwerbsminderung oder eine andere Aufbesserung des Haushaltseinkommens angewiesen. Nur das oberste Quintil der langjährigen ALGII-Bezieher mit noch lang andauernder Erwerbstätigkeit vor 2005 kommt mit $733 €$ in die Nähe der durchschnittlichen Grundsicherungsbeträge bei Erwerbsminderung.

\section{Fazit}

Die Absicherung der Langzeitarbeitslosen in der Gesetzlichen Rentenversicherung ist durch die Reform der Arbeitslosenhilfe zum Arbeitslosengeld II verschlechtert worden, denn der Bezug von Arbeitslosenhilfe führte bis $2000 \mathrm{zu}$ einer erheblich besseren Kompensation des Ausfalls von Beiträgen aus Erwerbstätigkeit in die Rentenversicherung. Auch wenn die Geldleistungen in der Arbeitslosenhilfe in den Jahren 2001 bis $2004 \mathrm{im}$ statistischen Durchschnitt nicht höher als spätere ALG-II-Leistungen waren, so wurden dennoch aufgrund der anderen Rechtslage mehr Beiträge an die Gesetzliche Rentenversicherung abgeführt. Die Einfüh- 
rung des ALG II steht damit für eine verringerte Absicherung in der Alterssicherung und bei Erwerbsminderung. Diese Gesamteinschätzung wird durch eine Ausnahme, die zu benennen ist, nicht durchbrochen: Nach Einführung des Arbeitslosengeldes II konnten vormalige Sozialhilfebezieherinnen und Sozialhilfebezieher - zumindest vorübergehend - erstmals Ansprüche in der Gesetzlichen Rentenversicherung erwerben, zu denen sie zuvor keinen Zugang hatten. Für diese Gruppe gilt, dass sie im Zuge der Reform die Voraussetzungen des Bezugs einer EM-Rente durch die Abführung von Pflichtbeiträgen aus ALG-II-Bezug an die Rentenversicherung erfüllen konnten. Diese Möglichkeit besteht seit 2011 allerdings nicht mehr und erschwert damit nachkommenden Gruppen von Langzeitarbeitslosen den Weg in die Erwerbsminderungsrente.

Lebenslaufanalysen mit Daten aus den Versicherungskonten haben gezeigt, dass in Westdeutschland $44 \%$ und in Ostdeutschland $7 \%$ der Versicherungsbiografien, die ALG-II-Bezug aufweisen, nur sehr geringe Anwartschaften in den Konten der Rentenversicherung erwerben. Bei Männern wie Frauen liegen die Gründe in schwerwiegenden und dauerhaften Erkrankungen bald nach Erwerbseintritt oder im mittleren Erwerbsalter. Weitere Fälle zeigen ein frühes Ausscheiden aus der sozialversicherungspflichtigen Beschäftigung - ohne Zeiten des Krankengeldbezugs. Bei Frauen tauchen in Westdeutschland außerdem Lebensläufe mit überdurchschnittlich langen Kinder-Erziehungszeiten und sehr wenig sozialversicherungspflichtiger Erwerbstätigkeit auf, die im späteren Leben in ALG II einmünden. Ostdeutsche Biografien sind weniger von deutlichen Lücken geprägt und weisen seltener langandauernde Zeiten des Krankengeldbezugs auf.

Zugänge in Erwerbsminderungsrente fanden in den letzten Jahren zu einem hohen Anteil aus dem langjährigen ALG-II-Bezug statt. Die Rentenhöhen sind dabei bei fast allen untersuchten Gruppen, die aus ALG II in die EM-Rente wechselten, zu niedrig, um eigenständig von dieser Rente leben zu können. Haushalte mit Erwerbsminderungsrentnern wurden inzwischen als ein von Armut gefährdeter Teil der Bevölkerung erkannt (Krause et al. 2013; Märtin et al. 2012). Die niedrigsten Renten haben erwartungsgemäß die EM-Rentnerinnen und -rentner, die vor der Einführung von ALG II nur sehr geringe Beschäftigungszeiten hatten und damit vor der Reform mit hoher Wahrscheinlichkeit die Voraussetzung der dreijährigen Beitragszeiten für eine Erwerbsminderungsrente gar nicht erfüllt hätten. In dieser Gruppe haben die Männer noch geringere Anwartschaften als die Frauen.

\section{LITERATUR}

Deutsche Rentenversicherung Bund (2006): Deutsche Rentenversicherung 9-10/2006, FDZ-RV, September/Oktober, Berlin

Deutsche Rentenversicherung Bund (2008): Die Versicherungskontenstichprobe als Scientific Use File. Workshop des Forschungsdatenzentrums der Rentenversicherung (FDZ-RV) am 30./31. Oktober in Würzburg, DRV-Schriften Bd. 79, Juli, Berlin
Deutsche Rentenversicherung Bund (2011): Die Renten wegen verminderter Erwerbsfähigkeit. Grundsätze der Deutschen Rentenversicherung, DRVSchriften Bd. 96, Dezember, Berlin

Knickrehm, S. (2008): Der Zwang zur Rente mit 63 gilt nicht für alle älteren ALG-II-Empfänger, in: Soziale Sicherheit, 57 (5), S. 192-198

Krause, P./Ehrlich, U./Möhring, K. (2013): Erwerbsminderungsrentner: Sinkende Leistungen und wachsende Einkommensunterschiede im Alter, DIW Wochenbericht 80 (24), S. 3-9

Märtin, S./Zollmann, P./Buschmann-Steinhage, R. (2012): Sozioökonomische Situation von Personen mit Erwerbsminderung. Projektbericht I zur Studie, DRV-Schriften Bd. 99, Oktober, Berlin

Mika,T./Lange, J. (2014): Auswirkungen der Einführung der Pflichtbeitragszeiten aus Arbeitslosengeld II auf den Rentenzugang wegen Erwerbsminderung und Alters, RVaktuell 2/2014 (im Erscheinen)

Stegmann, M./Werner, J./Müller, H. (2013): Sequenzmusteranalyse. Einführung in Theorie und Praxis, München/Mering

Thielen, K./Kroll, L. (2013): Alter, Berufsgruppen und psychisches Wohlbefinden, in: Bundesgesundheitsblatt 56 (3), S. 359-366

Trischler, F./Kistler, E. (2012): Gute Erwerbsbiographien: Internationales Institut für Empirische Sozialökonomie (inifes), Arbeitspapier 6: Was sind "gute" Erwerbsbiographien? Risikofaktoren und -gruppen für geringe Renten und Alterseinkünfte, Stadtbergen

Wübbeke, C. (2013): Ältere Arbeitslose am Scheideweg zwischen Erwerbsleben und Ruhestand: Gründe für ihren Rückzug vom Arbeitsmarkt, in: Journal for Labour Market Research 46 (1), S. 61-82

\section{AUTOREN}

TATJANA MIKA, Diplom-Soziologin und Juristin, ist Referatsleiterin im Geschäftsbereich Forschung und Entwicklung der Deutschen Rentenversicherung Bund. Arbeitsschwerpunkte: Erwerbsminderung, Migration, Alterssicherung.

tatjana.mika@drv-bund.de

JANINE LANGE, M.A. Soziologie, war Wissenschaftliche Mitarbeiterin am Forschungsdatenzentrum der Deutschen Rentenversicherung (FDZ-RV) in Berlin, derzeit Doktorandin an der Universität Rostock und Promotionsstipendiatin des „Forschungsnetzwerkes Alterssicherung“ (FNA) der Deutschen Rentenversicherung. Arbeitsschwerpunkte: Erwerbsminderung und Alterssicherung, soziale Ungleichheit, Lebenslaufanalyse.

janine.a.lange@drv-bund.de

MICHAEL STEGMANN, Dr., ist Referatsleiter im Geschäftsbereich Informationsverarbeitung der Deutschen Rentenversicherung Bund. Arbeitsschwerpunkte: Alterssicherung, Methoden der Längsschnittanalyse.

dr.michael.stegmann@drv-bund.de 